\title{
The Use and Promise of Conversational Agents in Digital Health
}

\author{
Tilman Dingler', Dominika Kwasnicka', Jing Wei', Enying Gong ${ }^{2}$, Brian Oldenburg ${ }^{2}$ \\ 1 NHMRC CRE in Digital Technology to Transform Chronic Disease Outcomes, School of Computing \\ and Information Systems, University of Melbourne, Parkville, Australia \\ 2 NHMRC CRE in Digital Technology to Transform Chronic Disease Outcomes, Melbourne School of \\ Population and Global Health, University of Melbourne, Melbourne, Australia
}

\begin{abstract}
Summary
Objectives: To describe the use and promise of conversational agents in digital health-including health promotion andprevention — and how they can be combined with other new technologies to provide healthcare at home.

Method: A narrative review of recent advances in technologies underpinning conversational agents and their use and potential for healthcare and improving health outcomes.

Results: By responding to written and spoken language, conversational agents present a versatile, natural user interface and have the potential to make their services and applications more widely accessible. Historically, conversational interfaces for health applications have focused mainly on mental health, but with an increase in affordable devices and the modernization of health services, conversational agents are becoming more widely deployed across the health system. We present our work on context-aware voice assistants capable of proactively engaging users and delivering health information and services. The proactive voice agents we deploy, allow us to conduct experience sampling in people's homes and to collect information about the contexts in which users are interacting with them.
\end{abstract}

Conclusion: In this article, we describe the state-of-the-art of these and other enabling technologies for speech and conversation and discuss ongoing research efforts to develop conversational agents that "live" with patients and customize their service offerings around their needs. These agents can function as 'digital companions' who will send reminders about medications and appointments, proactively check in to gather self-assessments, and follow up with patients on their treatment plans. Together with an unobtrusive and continuous collection of other health data, conversational agents can provide novel and deeply personalized access to digital health care, and they will continue to become an increasingly important part of the ecosystem for future healthcare delivery.

\section{Keywords}

Conversational agents, digital healthcare, telehealth, healthcare ecosystem

Yearb Med Inform 2021:191-9

http://dx.doi.org/10.1055/s-0041-1726510

\section{Introduction}

In 2020, telehealth experienced an unprecedented uptake around the globe, with the COVID-19 pandemic acting as a catalyst [1] Telehealth effectively reduces face-to-face consultations and, with it, the risk of viral exposure [1-3]. At the same time, Telehealth can maintain the delivery of many important health services and can increase the number of interactions that patients have with their health providers; thus, making the services more accessible and more cost-efficient to the patient and provider [1-5]. While the COVID-19 pandemic accelerated the introduction of virtual healthcare delivery in many countries, it also prompted the rapid development of many other diverse technology-enabled systems and processes for delivering virtual healthcare to patients [6]. For patients with mental health and/or chronic conditions requiring ongoing care and follow-up, COVID-19 has presented many challenges $[7,8]$.

One new technology development is the widespread uptake of conversational agents in people's lives, and these now also have many health applications [9]. Voice assis- tants, such as Google Home and Amazon's Alexa, have been widely adopted. In 2020, more than 83 million people in the U.S. use such 'smart' speakers, a $13.7 \%$ increased uptake compared to 2019. Conversational agents interact with their users using a text-based interface (i.e., chatbots) or a speech-based one (i.e., voice assistants). Commanded by users' language, they present a natural user interface and thus, can make their services and applications accessible to a wide range of the population [10].

The functionality of conversational agents ranges from triggering notifications to responding to simple commands and questions [11]. More sophisticated versions retain the progression and context of a conversation across multiple sessions, which allows them to provide highly customized interactions. As more and more sensors and smart devices are being used in consumers' homes as part of the context of the Internet of Things, this is creating a new ecosystem of technology-enabled devices and services [12]. Conversational agents offer an intuitive interface in this ecosystem between the user, the user's data, and their other home appliances and devices.

In this article, we discuss the potential of these trends being enabled by conversational agents to seamlessly integrate user needs, data, and health services. Further, by tapping into personal health data that are available from wearable devices, such as Fitbit and Apple Health, these smart agents can also trigger tailored conversations about people's health states and can also triage support to be delivered by other health services. We describe how such exchanges of information 
about a person's health and related issues can become a continuous, seamless "conversation" between patients, their data, and their health providers. The delivery of health services in this way can be personalized to a person's specific needs and preferences. Furthermore, the semi-automated nature of the conversation can relieve some of the pressures from the healthcare system. This approach can be scalable, and people can access health services 24 hours/7 days a week and from any location. We also present some of our own work on context-aware voice assistants that are capable of proactively engaging users and delivering health information and services.

We begin with a brief history of the origins of conversational agents to explain common shortcomings that lead us to enhance commercially available voice agents with proactive features. We then present common usages of conversational agents in healthcare, as evidenced by related work. From here, we draw a picture of the future applications of interconnected health services to which conversational agents can provide the user interface in the form of ongoing, highly personalized conversations about the user's health and well-being.

\section{Conversational Agents: Evolution in Personalization}

The idea to converse with computers using natural language goes back to Alan Turing's seminal Imitation Game [13]. He devised what is today known as the Turing Test, which challenges people to determine whether they are talking to another human or, in fact, a machine. A machine passes the test when the distinction is no longer clear. In 1966, Joseph Weizenbaum developed ELIZA, a chatbot, which he originally created to demonstrate how limited the communication between humans and machines were at the time. Using simple pattern matching and substitutions, the chatbot conveyed the mere illusion of understanding its users. The chatbot took the form of a Rogerian psychotherapist named after Carl Rogers, using his famous method that involved slightly rephrasing and repeating what patients had just said. And while Weizenbaum ended up being surprised by users' tendency to attribute human-like feelings to the chatbot, today's systems and devices are often seen as companions and human-like entities with social elements and personalities [14]. Personality traits are increasingly being added to conversational interfaces to build trust with users [10] by adapting to users' personal preferences [15]. Subsequently, in 1995, Richard Wallace created the Artificial Linguistic Internet Computer Entity (ALICE), an award-winning chatbot capable of processing natural language and engaging in conversations with humans using pattern-matching [16]. Nowadays, conversational agents are widely deployed in the form of chatbots supporting customers with bookings, helping with banking tasks, and answering frequently asked questions.

Early chatbots followed static scripts and based on the user response, would choose the most fitting continuation of the conversation. This pattern reflects current online question-answer assistants where users ask questions, and the agent triggers a response based on simple pattern matching. Such chatbots are most widely deployed as their implementation does not require specific programming knowledge and is rather based on tools, such as Google's Dialogflow [17]. More sophisticated agents store and retrieve user information in databases and personalise the conversation around the user's profile. A profile can consist of personal data, such as name and age, of interaction history, i.e., information gathered in previous conversations, and context, such as time of day, the medium through which the user converses, location, or device proximity. The more user data is available, the more the agent can adjust the conversation around the user's goals and needs.

Types of conversational agents range from dialogue systems (e.g., a common system used by hospitals to triage incoming phone calls regarding severity, urgency, and pathology) to previously mentioned online chatbots and voice assistant. Sometimes, agents come with human-like avatars to signal trust and connectedness. These embodied agents are often used on desktop computers and web applications and have been developed for a range of different conditions [18]. Voice assistants communicate specifically through spoken language and can but do not have to come with a visual representation. Amazon Alexa, Google Home, Apple's Siri, and Microsoft's Cortana are the most prominent and widely used voice agents [19], and they do not have associated visual representations. In the case of Siri and Cortana, they are shipped out with the phone's operating system. Alexa and Google Home can be controlled through the phone as well but also reside in special hardware devices at users' homes.

\subsection{Towards Proactive Conversational Agents}

Voice assistants constantly listen for user commands and thus currently, play a relatively passive role. To initiate a conversation, Alexa can light up an LED ring to signal a notification, which then needs to be actively received and answered by the user. However, other more proactive features are still missing. Activation at inconvenient times can quickly be perceived as intrusive and annoying [20]. Proactive agents are currently being researched and developed in two ways: as 1) a context-aware proactive voice assistant; and 2) as autonomous agents acting on behalf of their user.

\subsection{Context-aware Voice Assistants}

Google Home and Amazon Echo are currently limited to sending simple notifications or setting off alarms. Such interactions merely transfer smartphone's notifications to speakers and usually, do not employ a back-and-forth multi-turn-taking interaction with the user. To enable more fluent human-like conversations, however, conversations agents need to be able to initiate conversations and engage users in longer turn-taking conversations [21, 22]. Compared with notifications on smartphones, which can be silenced or limited to vibrations, voice notifications from conversational agents are more interruptive as 
voice or audio are more difficult to ignore. Users may only engage in conversations with the conversational agent if the agent initiates the conversation at an opportune moment. Conversational agents, which can conduct bi-directional conversations, need, therefore, to become aware of the user's current context so that they can detect appropriate times for initiating conversations.

In our research, we investigate under which circumstances smart speakers can trigger interactions with their users based on contextual factors [23]. These factors include device location, time of day, user proximity, ambient light levels and current noise levels. Figure 1 shows our current prototype, which consists of a Google Home assistant we modified. Two earphones attached to the speaker can deliver pre-recorded voice commands inaudible to the user. Those pre-recorded voice commands invoke our custom Google Action (voice applications). This setup allows us to run experience sampling surveys, which provide subjective user assessments throughout the day [24]. Our current custom Google Action implements a 4-item mini-survey. To gauge the users' current context, the speaker asks about people's availability, boredom level, mood, and current activities. Invoking the survey is done in regular intervals but, with the help of sensor data, surveys can be triggered by certain events as well, such as the presence of a person, when the user wakes up in the morning or before leaving their home. For patients living with chronic health conditions, specific types of mini-surveys and reminders can be implemented in voice applications and be deployed on our system to collect data about patients' medical or mental conditions and support medication adherence. As this system is deployed in people's home, we can, therefore, study how proactive conversational agents can adapt to the needs of patients with different chronic conditions in their daily life. The system can learn when patients are more available and receptive to voice-based interactions with conversational agents under different contexts. Thus, just-in-time data collection or interventions [25] can be delivered through context-aware conversational agents.

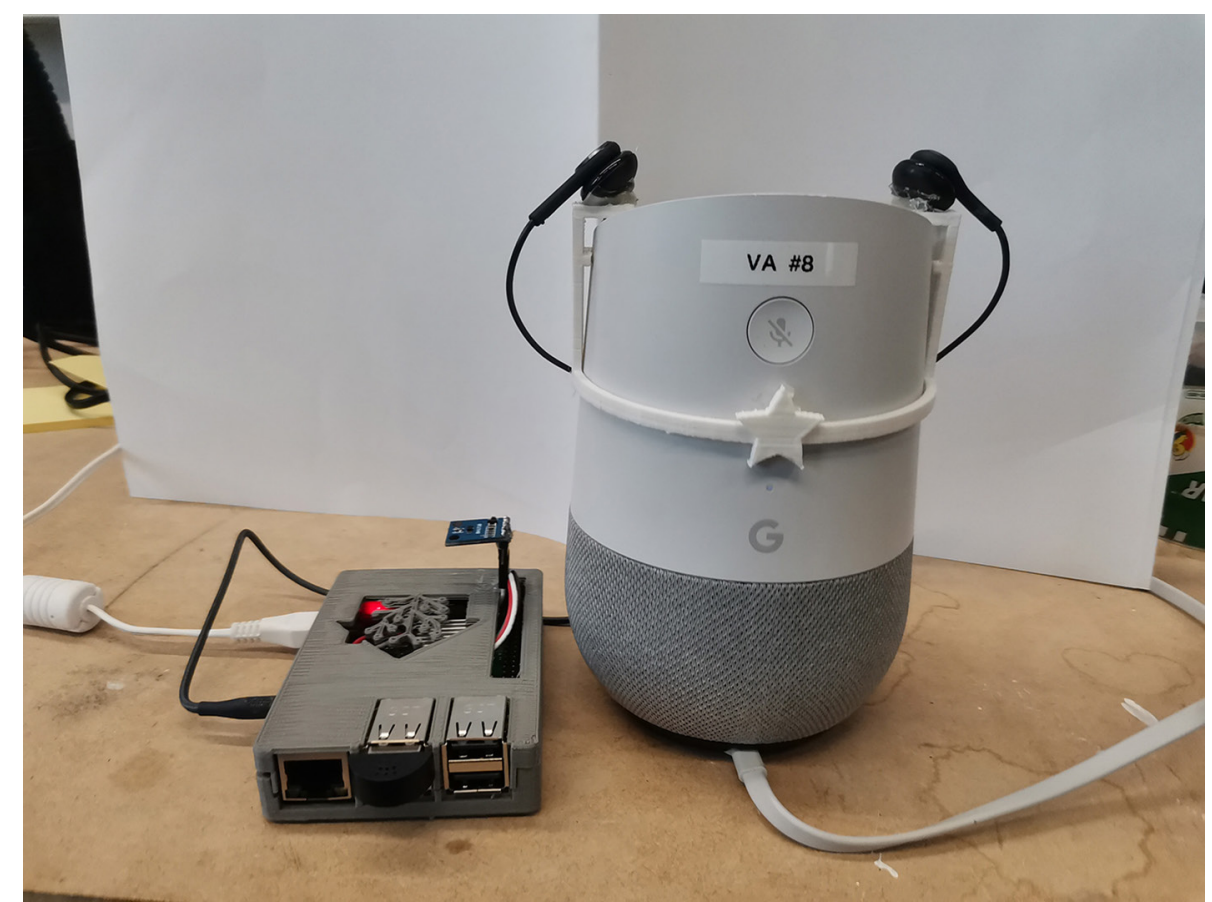

Fig. 1 A proactive Google Home assistant. We attached earphones to the speaker, which deliver inaudible sound bites for activating the device and invoking custom applications, such as triggering a health survey or invoking a medication reminder.

\subsection{Autonomous Agents}

The second type of active agents is a fully autonomous system acting on behalf of its users. In 2018, Google CEO Sundar Pichai introduced a new Google assistant, which booked an appointment at a hair salon via phone for its user [26]. Using speech synthesis with natural language elements, the system was close to indistinguishable to a human voice over the phone. The system called Google Duplex is the first example of a conversational agent that is, indeed, a personal assistant to its user [27]. For people living with chronic conditions, or indeed, people experiencing a more acute illness, the ability of an agent to book a doctor's appointment or call an ambulance could be lifesaving. We expect to see more of such autonomous systems being commonly used in the future as more health services expand their online offerings and provide online bookings.

\section{Application and Evidence of Conversational Agents in Healthcare}

Conversational agents are being increasingly applied in the healthcare field as a promising tool to improve delivery and quality, stimulated in part by the advent of COVID-19. Many observations have been made regarding the potential of conversational agents in addressing the temporal, geographical and organizational barriers $[28,29]$. Patients and healthcare professionals have also demonstrated a general acceptance of conversational agents and high perceived usefulness, convenience and engagement in overcoming logistical and service barriers. However, studies have also raised concerns regarding the quality of information, responsiveness, and potential ethical risks have also been 
raised [28-30]. Errors may occur when the conversational agents misrecognize users' input; the users express an intent that the system cannot handle, or the system provides incomplete or inaccurate information to users. Many of these errors are quite common for non-health domains, but they may bring significant harm to patients if the conversations are about medicine use or emergencies. A multi-layered escalation from a conversational agent to connecting to a health professional is, therefore, required. In addition to the program errors, conversational agents may also introduce biases and ethical challenges if the sampling of the data sets used to train the algorithms were biased to particular racial or ethnic groups or the prediction models are not disproportionately accurate for certain subgroups of consumers.

Several recent review articles have described the scope of conversational agents in healthcare. Laranjo and colleagues identified 17 conversational agents with unconstrained natural language input capabilities in healthcare. They found that conversational agents in healthcare are still in the early stage compared to other fields [31]. Carr et al. updated the review and yielded 47 studies covering all types of conversational agents in healthcare, indicating a rising trend of studies in this field since 2016 [32]. This recent scoping review also found that about half of the identified agents were delivered via smartphone apps or smartphone-embedded software as the major delivery channels, followed by web-based chatbots. Conversational agents are also increasingly designed to increase the program's engagement and personalization, either as an authority and knowledgeable identity as a virtual health professional or coach or as an informal human-like friend and peer $[32,33]$.

Other reviews have undertaken a content analysis of conversational agents in healthcare. Conversational agents have been developed to support patients and healthcare professionals in specific tasks; thus, a large proportion of conversational agents in healthcare have been considered as goal-oriented agents [32]. The health goals of conversational agents can be as short as answering immediate health-related queries or they can be much more long-term as part of building a relationship with the users in order to provide personalized education, coaching and monitoring. One recent review developed a taxonomy for conversational agents in health. It classified the health purpose of conversational agents into six groups: training, education, assistance, prevention, diagnosis, and elderly assistance [34]. Car and colleagues also described a shifting trend in health goals of conversational agents from monitoring and tracking, to connecting patients to health care services and supporting users in achieving long-term behaviour change goals and chronic disease management [32].

- Screening, diagnosis and triage: Several conversational agents, mainly health chatbots, have been developed for screening patients with cancers, mental health disorders and risk of chronic diseases [35-38]. Some chatbots were developed following the finite-state dialogue management system. The agents controlled the conversation flow and contained structured questions and scripts with symptom checklists or scales to assess the risk of diseases. During the COVID-19 pandemic, an increasing number of health chatbots were released based on mobile communication apps such as WhatsApp, LINE, WeChat to assist self-triage and personal risk assessment [39]. Besides, empowered by AI, the screening and triage system could fulfil more complex requirements to achieve automated triage. For example, the Babylon AI-powered Triage and Diagnostic system allows people to be automatically triaged as their first contact point with the healthcare system before linking with GPs or specialists. The Babylon chatbots offer AI-empowered triage based on users' self-reported symptoms, personal medical history and a medical knowledge database reviewed by clinical experts. The chatbots triage users and provide them with an action plan to link further appointment-making with GPs or specialists [40]. Studies have shown its general safety and accuracy compared with human doctors, which indicated a great potential in saving health workforce and resources [41].
- Treatment assistant, disease management and consultations: Conversational agents could provide consultations to empower patients to engage more fully before and during the clinical appointment. The conversational agents were developed by mimicking human interaction between healthcare providers and patients using natural language processing and machine learning. By exchanging information and providing live responses, conversational agents may collect patients' information before their appointment to provide tailored counselling and improve the quality of visits [42]. Conversational agents are increasingly applied in the field of mental health. Provoost and colleagues reviewed studies that used embodied conversational agents in clinical psychology and found that conversational agents were used for social skills training, cognitive behaviour therapy, and counselling for depression and anxiety, though more than half of identified studies were focused on autism treatment [43].

- Assisting self-management and behaviour change: Pereira and colleagues reviewed studies that used health chatbots for behaviour change $[44,45]$. They emphasized the role of chatbots in monitoring bio-signals, coaching and advising users by improving their self-efficacy and cognition, providing encouragement and reinforcement through mood analysis and influencing patients' behaviour through distraction and encouragement. These approaches and behaviour change techniques were applied to promote patients' self-management in chronic conditions, mental and neurological disorders and addictions

- Training and education: The purpose of training and education is achieved mainly by providing information to users via conversations and coaching users to acquire skills. An increasing number of studies have applied virtual coaches or virtual nurses via an Avatar, embodied conversational agents to provide personalised coaching for people with chronic diseases and healthy elderly [46]. In addition, virtual patients have also been developed to simulate real clinical scenarios to train medical students in performing physical 
exams and clinical judgment to build medical students' capacity before they perform clinical practice [46].

Despite the increasing use of conversational agents in healthcare, the evidence on the efficacy and effectiveness of conversational agents in improving health and well-being is still mostly lacking and piecemeal. MilneIves and colleagues conducted a review on the general effectiveness of AI-empowered conversational agents in healthcare through synthesizing findings from experiments and trials. They found that only three-quarters out of 31 identified studies reported positive or mixed evidence of effectiveness in clinical or behavioural outcomes, and only five studies evaluated the cost-effectiveness of the program. Studies with robust design and comprehensive evaluation are desperately needed to provide evidence before scaling up the innovations into the real-world setting (47).

\subsection{Continuous Access to Health Services}

In our current work at the NHMRC Centre for Research Excellence in Digital Technology to Transform Chronic Disease Outcomes, we are researching and developing proactive conversational agents with the goal to make healthcare services more personalized and easier to navigate. For instance, our recent trial tested and showed that conversational agent 'Laura' could support people living with type II diabetes [48]. The agent provides brief interactions to support the person with diabetes in goal setting, action planning and then, following up and asking relevant questions about progress with the self-management of diabetes. Conversational agents, such as 'Laura' can have multiple uses in supporting people who are living with chronic conditions. They can even provide emotional and social support, use effective behaviour change techniques to improve current health behaviours, such as physical activity, healthy eating, medication adherence [49]. In addition to supporting individuals, conversational agents may also save the healthcare system costs by reducing unnecessary hospital attendance and emergency department visits [50].
In another project, we designed and implemented agents that are part of a smart home environment of people living with chronic heart failure [51]. Up to two-thirds of heart failure hospitalizations are preventable as indicated by hospital data [52]. Supporting self-management of this condition is the focus of the strand of conversational agents we are currently developing. They help patients monitor their symptoms, adhere to medication plans, stick to diet and exercise regimens and manage symptoms by recognizing symptom changes and initiating measures, such as behaviour change or reaching out to attain appropriate assistance.

Over the last five years, chatbots have entered mainstream messaging services, such as Facebook Messenger and WhatsApp. These bots autonomously chat with their users inside the messaging app itself without the need to install another application. They "live" right next to private conversations users have with friends and family providing easy access and lowering the threshold to interact. As most users interact with messaging apps several times a day [53], chatbot conversations are of high visibility. For older phone versions that do not support a modern app marketplace, conversational agents can communicate through conventional SMS as well. Depending on the available infrastructure, agents are, therefore, available in rural regions providing access to health service to people across the geographical and economic spectrum.

Easy access to and the ability to keep track of patients' conversations and data allows these agents to personalize the information and information delivery to an unprecedented degree. If the agent has access to the patient's clinical and health services history and, once authorized, the system does not need to repeatedly request patients' credentials as is the case with current consultations over the phone. This can save considerable time and conveys the idea to the patient of having a personal health coach literally "in their pocket". Often anthropomorphic elements, such as a human-like avatar or natural language use, make interactions more humane and personal. By incorporating personality into conversational agents and emotional aspects into dialogues, the agents are also capable of building trust and rapport with patients.
As little real human intervention is needed for triaging health services, booking appointments, sending medication reminders, or following up on the development of symptoms, conversational agents are highly scalable as their ability to converse with an entire patient population is virtually unlimited. Communication can happen asynchronously, meaning patients can request health service information and support at any time with a conversational agent, without the need to wait for a dedicated connection or appointment with a health provider unless there is a need to do so. Agents' support, therefore, is immediate as they issue prompt responses. Triaging services is objective, and assessments are generally unbiased during the interaction. However, biases can be introduced in other ways, for example, during the training of the language model or of the recommendation algorithms. Special care needs to be applied to make sure that training data is as unbiased as possible, an active field for research around the ethics of artificial intelligence.

While the goal of personalization is to create a more personal and customized relationship between patient and health care services, conversational agents can also offer the safety of anonymity [11]. Users will sometimes feel more comfortable interacting with an "anonymous" conversation agent compared to a healthcare professional [54]. Stigma and barriers to healthcare advice access can be significantly reduced, and that can then allow exploration of several challenging healthcare topics [55]. This is particularly relevant for patients seeking mental health support and explains why the uptake has been so impressive in this regard.

As evident by the examples given, conversational agents can also handle a range of highly sensitive data. Hence, they need to be designed in a way that supports and guarantees patients' privacy and data security. We recommend following tried and tested protection strategies as they are employed in other web and mobile technologies, including the use of login credentials, two-factor authentication (i.e., patients need to verify their identity through two separate channels), biometric authentication (e.g., fingerprint, facial recognition), and data encryption techniques. While the exchange of some 
patient data is indispensable to integrate data sources and communicate patient states to health care professionals, other data can be collected, processed, and stored on the patients' local devices.

Such privacy-by-design architecture can enable appropriate compromises being made between data integration, privacy, and ownership. Another compromise is usually struck at the interface level, where usability and security requirements collide. A secure system is safe from password guessing or impersonation attempts. Usable security research looks at how to make systems both safe and user-friendly. To make conversational agents accessible to a broad population, they need to be easy to set up yet safe to use. Home assistants can employ voice recognition technology to authenticate users based on their voice and speech patterns, for example, even in a household with several people. We, thereby, need to consider the delicate interplay between household residents, conversational agents, and the home and wearable devices.

\subsection{The Interconnected Interface}

Effective patient monitoring includes continuous, unobtrusive data collection with explicit check-ins. Creating a technology-enabled healthcare ecosystem can be understood as a multilateral conversation between users, their data, and healthcare services. To ensure continuous information exchange, conversational agents need to be able to initiate and actively undertake such conversations. Chatbots can trigger conversations using notifications and sending reminders in chat based on time intervals. Event-triggered reminders, on the other hand, can be based on contextual information, such as the number of steps taken. Current smart speakers, such as Google Home or Amazon's Alexa, usually behave in a passive way; however, they respond to activation commands (e.g., "Hey Google") explicitly initiated by the user. Users, therefore, need to actively invoke current smart speakers to request data (e.g., steps) or to report events (e.g., have taken medications). Proactive smart speakers, in contrast, can provide a voice interface to actively inform patients of useful health data collected from wearable and IoT devices such as steps, calories burned, and heart rate or provide them with necessary and evidence-based advice and interventions based on in-situ or long-term monitoring of patients' health states.

To create such an ecosystem to fully support and enable patients to manage complex chronic conditions at home will benefit from an interconnected environment, a network of smart devices. The Internet of Things (IoT) describes the vision of such a network of connected devices that includes sensors, software, services, and output modalities. Access to sensor and device information in patients' homes will allow the connected agent to make inferences about the user state and context, including the person's health condition and circumstances. Third-party data streams from devices, such as Fitbit or the Apple Watch, can also be accessed and integrated to provide a more holistic picture. The agent provides the interface for continuous engagement: an ongoing conversation between the user, user data, and health services.

\subsection{Enabling Technologies for Healthcare in the Home}

Software disciplines, such as natural language processing (NLP), have made significant leaps in recent decades. Throughout most of the late $20^{\text {th }}$ century, NLP software was mainly rule-based when in the 1990's, machine-learning techniques were used to statistically process natural language. With advances in machine learning and the eventual switch to neural networks, NLP started becoming on par with transcriptions by humans. These advances paved the way for commercial voice assistants, such as Amazon Alexa (introduced in 2012) and Google Nest (released in 2014).

Conversational agents with their natural user interface have the potential to become the primary user interface for text- and voicebased interactions with apps and services. New tools and development frameworks make it possible to create agents without much domain expertise in machine learning. Further, there is a range of open-source frameworks, such as RASA [56], which build a starting point to create custom agents. This variant provides a higher degree of customization and flexibility but requires a certain expertise and development resources. And while the market and its technologies are still relatively young, there is much innovation, product development, and research around bringing smart conversational agents to people with the goal to support health and well-being.

As speech recognition becomes more sophisticated and human-like, commercial voice assistants increasingly enter people's homes, and the ecosystem of tools to build conversational agents grows, we anticipate a steep proliferation of health applications and services in the next years, opening the way for more digital innovations in healthcare. In the last Section, we will outline some scenarios in which conversational agents could become the primary interface between patients and health services.

\subsection{Triaging Health Issues}

There is already a range of dedicated health apps, such as MyDiabetesCoach [57] or the Woebot [58] which is a digital mental health therapist that provides self-management support. Yet, we see the biggest potential of conversational agents in their ability to help patients effectively navigate health services, connect to health resources, and engage in ongoing conversations about health and well-being. The aim of conversational agents is, therefore, not to replace health professionals but rather, to help patients to more effectively navigate the health system and to empower them to better manage their own health. This should save time and money on both sides: patients and health professionals. Additionally, conversational agents may support healthcare professionals in healthcare provision, e.g., by providing patients with relevant reminders, booking their clinical appointments, allowing them to contact healthcare professional via telehealth consultations, supporting efficient information exchange with hospital information system (such as HIS, PACS and CIS), and providing regular checking and monitoring at large scale. 
The Use and Promise of Conversational Agents in Digital Health

For example, let us consider the example of symptom tracking of active COVID-19 cases, which requires monitoring by having medical professionals regularly checking in for symptoms development. The sheer number of active cases may already be overwhelming for a regional hospital but monitoring active cases only may not be sufficient. For effective COVID tracing, the broader circle of people who have been in contact with active cases need to be monitored as well. Therefore, the number of people who require regular check-ins increases exponentially as the circle of contacts increases and this makes manual tracking by medical professionals (or other service providers) almost impossible.

Automated systems can send out surveys and reminders to ask people to report symptoms, but other than email, solid technology infrastructure is missing to coordinate these, store the data, and follow-up with missed reports. Chatbots can help here as they can 'live' in people's messaging services, such as WhatsApp, Facebook Messenger, or Apple's iMessage. They are easily subscribed to by sending a simple welcome message and remind their users to frequently answer questions about their possible symptoms and report if any new symptoms develop over time. Figure 2 depicts the information flow between patients and health care services with the conversational agents triaging most of the traffic. In case symptoms continue to deteriorate over time, the chatbot either recommends contacting local healthcare services or proactively forwards the user's contact to be reached out to. Dialogue systems have long been in use, triaging patients' calling hospitals, but the sheer number of triage services makes the system slow and often causes patient frustration [59].

In our work, we design and develop conversational agents that support health care services, scale their accessibility, and install a rigorous check-in regime to ensure the collection of frequent data points and retain the connectedness between health institutions and patients. Figure 2 shows how our conversational agent 'lives' on patients' devices and acts as starting point for checking their health state or requesting health services. The agent provides highly customized recommendations, stores and integrates the patient's various data points from other sources, such as wearable technologies, and proactively checks on patients' symptoms and health. It shares data where necessary with the patient's GP and autonomously books appointments when needed. Once the patient explicitly grants the GP data access, the patient's state can be inspected at any point in time using a data dashboard. In the case of deteriorating or ambiguous health conditions, the GP can take over the conversation and initiate a personal chat or phone call. Health will be a continuous conversation between patients, caretakers, and their data, facilitated by conversational agents.

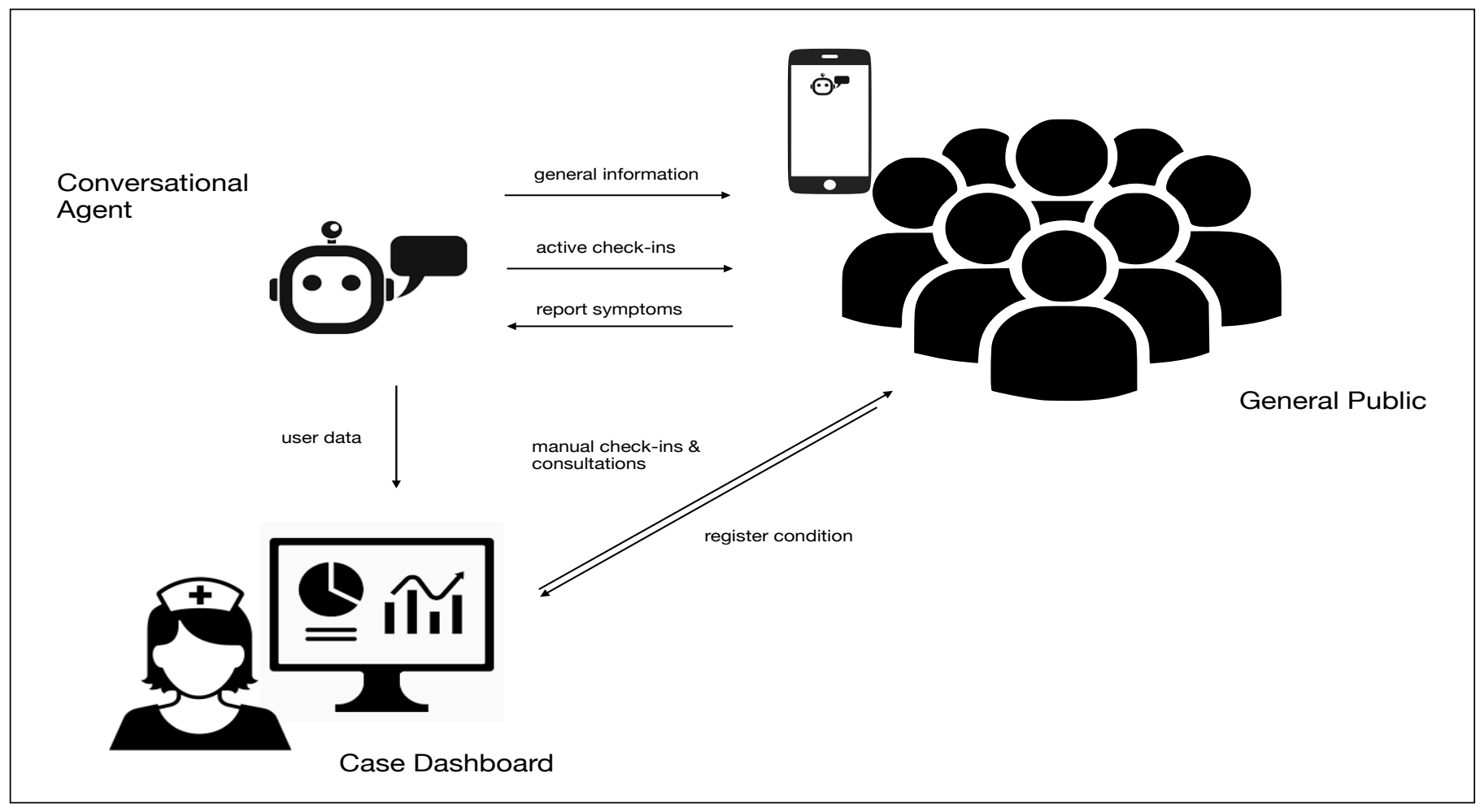

Fig. 2 Conversational agents effectively triage patient requests while customizing their support around the patient's personal information and interaction history. 


\section{Conclusion}

The use of conversational agents is slowly becoming more common in healthcare delivery. These agents have a variety of essential characteristics, which make them an increasing focus of study and commercial interest in digital health and healthcare delivery. Conversational agents can be widely accessible and highly personalised health "companions". They can be accessed on a person's phone, through a website visit on a desktop computer, or via a dedicated device, such as Amazon Alexa. The now almost ubiquitous availability of mobile devices and recent commercialization of smart speakers make these agents more widely accessible. With language and speech being natural interaction modalities, this enhances the intuitiveness of their use and makes them easy to learn. While chatbots are traditionally text-based, voice assistants use speech to interact with their users. The use and promise of conversational agents in the healthcare ecosystem is rapidly increasing. All the advantages notwithstanding, researchers, users and technology developers also do need to be aware of the extra layers of complexity in terms of privacy, security and data access when using conversational agents in healthcare settings.

\section{Acknowledgements}

The NHMRC Centre of Research Excellence (CRE) in Digital Technology to Transform Chronic Disease Outcomes aims to transform Australia's digital health ecosystem. It is funded by the NHMRC (2020-2024: ID 1170937) and involves researchers from the University of Melbourne, Deakin University, Griffith University and Macquarie University in Australia and Palo Alto and Stanford Universities in USA. The centre is leveraging the ubiquitous availability of smart devices and digital technology to improve the health and wellbeing of the population, particularly those with chronic conditions.

\section{References}

1. Wosik J, Fudim M, Cameron B, Gellad ZF, Cho A, Phinney D, et al. Telehealth Transformation: COVID-19 and the rise of Virtual Care. J Am Med Inform Assoc 2020;27(6):957-62.
2. Monaghesh E, Hajizadeh A. The role of telehealth during COVID-19 outbreak: a systematic review based on current evidence. BMC Public Health 2020;20(1):1-9.

3. Rush KL, Hatt L, Janke R, Burton L, Ferrier M, Tetrault M. The efficacy of telehealth delivered educational approaches for patients with chronic diseases: A systematic review. Patient Educ Couns 2018;101(8):1310-21.

4. Jin K, Khonsari S, Gallagher R, Gallagher P, Clark AM, Freedman B, et al. Telehealth interventions for the secondary prevention of coronary heart disease: a systematic review and meta-analysis. Eur J Cardiovasc Nurs 2019;18(4):260-71.

5. Orlando JF, Beard M, Kumar S. Systematic review of patient and caregivers' satisfaction with telehealth videoconferencing as a mode of service delivery in managing patients' health. PloS One 2019;14(8):e0221848.

6. Chudasama YV, Gillies CL, Zaccardi F, Coles B, Davies MJ, Seidu S, et al. Impact of COVID-19 on routine care for chronic diseases: a global survey of views from healthcare professionals. Diabetes Metab Syndr Clin Res Rev 2020;14(5):965-7.

7. Wright A, Salazar A, Mirica M, Volk LA, Schiff GD. The invisible epidemic: neglected chronic disease management during COVID-19. J Gen Intern Med 2020;35(9):2816-7.

8. Druss BG. Addressing the COVID-19 pandemic in populations with serious mental illness. JAMA Psychiatry 2020;77(9):891-2.

9. Sezgin E, Huang Y, Ramtekkar U, Lin S. Readiness for voice assistants to support healthcare delivery during a health crisis and pandemic. Npj Digit Med 2020;3(1):1-4.

10. Braun M, Mainz A, Chadowitz R, Pfleging B, Alt F. At Your Service: Designing Voice Assistant Personalities to Improve Automotive User Interfaces. In: Proceedings of the 2019 CHI Conference on Human Factors in Computing Systems [Internet]. Glasgow Scotland Uk: ACM; 2019 [cited 2021 Feb 2]. p. 1-11. Available from: https://dl.acm. org/doi/10.1145/3290605.3300270

11. Laranjo L, Dunn AG, Tong HL, Kocaballi AB, Chen J, Bashir R, et al. Conversational agents in healthcare: a systematic review. J Am Med Inform Assoc 2018;25(9):1248-58.

12. Singh RP, Javaid M, Haleem A, Suman R. Internet of things (IoT) applications to fight against COVID-19 pandemic. Diabetes Metab Syndr Clin Res Rev 2020;14(4):521-4.

13. Machinery C. Computing machinery and intelligence-AM Turing. Mind 1950;59(236):433.

14. Reeves B, Nass C. The media equation: How people treat computers, television, and new media like real people. Cambridge university press Cambridge, UK; 1996.

15. Mairesse F, Walker MA. Towards personality-based user adaptation: psychologically informed stylistic language generation. User Model User-Adapt Interact 2010;20(3):227-78.

16. Wallace RS. The anatomy of ALICE. In: Parsing the Turing Test. Springer; 2009. p. 181-210.

17. Dialogflow Google Cloud [Internet]. [cited 2021 Feb 2]. Available from: https://cloud.google.com/ dialogflow/
18. Gong E, Baptista S, Russell A, Scuffham P, Riddell M, Speight J, et al. My Diabetes Coach, a Mobile App-Based Interactive Conversational Agent to Support Type 2 Diabetes Self-Management: Randomized Effectiveness-Implementation Trial. J Med Internet Res 2020 Nov 5;22(11):e20322.

19. Kepuska V, Bohouta G. Next-generation of virtual personal assistants (microsoft cortana, apple siri, amazon alexa and google home). In IEEE; 2018. p. 99-103.

20. Park C, Lim J, Kim J, Lee S-J, Lee D. Don't Bother Me. I'm Socializing! A Breakpoint-Based Smartphone Notification System. In: CSCW 2017. Proceedings of the 2017 ACM Conference on Computer Supported Cooperative Work and Social Computing; 2017. p. 541-54.

21. Sacks H, Schegloff EA, Jefferson G. A simplest systematics for the organization of turn taking for conversation. In: Studies in the organization of conversational interaction. Elsevier; 1978. p. 7-55.

22. Liao QV, Davis M, Geyer W, Muller M, Shami NS. What can you do? Studying social-agent orientation and agent proactive interactions with an agent for employees. In: DIS '16. Proceedings of the 2016 ACM Conference on Designing Interactive Systems; 2016. p. 264-75.

23. Wei, J., Dingler, T. and Kostakos, V. Developing the Proactive Speaker Prototype Based on Google Home. In: Extended Abstracts of the $2021 \mathrm{CHI}$ Conference on Human Factors in Computing Systems; 2021. p. 1-6.

24. Consolvo S, Walker M. Using the experience sampling method to evaluate ubicomp applications. IEEE Pervasive Comput 2003;2(2):24-31

25. Nahum-Shani I, Hekler EB, Spruijt-Metz D. Building health behavior models to guide the development of just-in-time adaptive interventions: A pragmatic framework. Health Psychol 2015;34(S):1209.

26. Google Duplex: A.I. Assistant Calls Local Businesses To Make Appointments - YouTube [Internet]. [cited 2021 Feb 2]. Available from: https://www.youtube.com/atch?v=D5VN56jQMWM\&t=3s\&ab_channel=JeffreyGrubb

27. Hancock JT, Naaman M, Levy K. AI-mediated communication: definition, research agenda, and ethical considerations. J Comput-Mediat Commun 2020;25(1):89-100.

28. Palanica A, Flaschner P, Thommandram A, Li M, Fossat Y. Physicians' perceptions of chatbots in health care: Cross-sectional web-based survey. J Med Internet Res 2019;21(4):e12887.

29. Househ M, Doha R. Perceptions and Opinions of Patients About Mental Health Chatbots: Scoping Review. J Med Internet Res 2021;23(1):e17828.

30. Luxton DD. Ethical implications of conversational agents in global public health. Bull World Health Organ 2020;98(4):285.

31. Laranjo L, Dunn AG, Tong HL, Kocaballi AB, Chen J, Bashir R, et al. Conversational agents in healthcare: a systematic review. J Am Med Inform Assoc 2018;25(9):1248-58.

32. Car LT, Dhinagaran DA, Kyaw BM, Kowatsch T, Rayhan JS, Theng YL, et al. Conversational agents in health care: Scoping review and conceptual analysis. J Med Internet Res 2020;22(8):e17158.

33. Kocaballi AB, Berkovsky S, Quiroz JC, Laranjo L, Tong HL, Rezazadegan D, et al. The per- 
sonalization of conversational agents in health care: systematic review. J Med Internet Res 2019;21(11):e15360.

34. Montenegro JLZ, da Costa CA, da Rosa Righi R. Survey of conversational agents in health. Expert Syst Appl 2019;129:56-67.

35. Chang P, Sheng Y-H, Sang Y-Y, Wang D-W. Developing a wireless speech-and touch-based intelligent comprehensive triage support system. CIN Comput Inform Nurs 2008;26(1):31-8.

36. Xu R, Mei G, Zhang G, Gao P, Judkins T, Cannizzaro $\mathrm{M}$, et al. A voice-based automated system for PTSD screening and monitoring. Stud Health Technol Inform 2012;173:552-8.

37. Spänig S, Emberger-Klein A, Sowa J-P, Canbay A, Menrad K, Heider D. The virtual doctor: An interactive clinical-decision-support system based on deep learning for non-invasive prediction of diabetes. Artif Intell Med 2019;100:101706.

38. Ghosh S, Bhatia S, Bhatia A. Quro: facilitating user symptom check using a personalised chatbot-oriented dialogue system. Stud Health Technol Inf 2018;252:51-6.

39. Almalki M, Azeez F. Health Chatbots for Fighting COVID-19: a Scoping Review. Acta Inform Medica 2020;28(4):241.

40. Middleton K, Butt M, Hammerla N, Hamblin S, Mehta K, Parsa A. Sorting out symptoms: design and evaluation of the'babylon check'automated triage system. ArXiv Prepr ArXiv160602041.2016.

41. Razzaki S, Baker A, Perov Y, Middleton K, Baxter J, Mullarkey D, et al. A comparative study of artificial intelligence and human doctors for the purpose of triage and diagnosis. ArXiv Prepr ArXiv180610698. 2018.

42. Adams WG, Phillips BD, Bacic JD, Walsh KE, Shanahan CW, Paasche-Orlow MK. Automated conversation system before pediatric primary care visits: a randomized trial. Pediatrics 2014;134(3):e691-9.

43. Provoost S, Lau HM, Ruwaard J, Riper H. Embodied conversational agents in clinical psychology: a scoping review. J Med Internet Res 2017;19(5):e151

44. Pereira J, Díaz Ó. Using health chatbots for behavior change: A mapping Study. J Med Syst 2019;43(5):135.

45. Williams ED, Bird D, Forbes AW, Russell A, Ash $S$, Friedman R, et al. Randomised controlled trial of an automated, interactive telephone intervention (TLC Diabetes) to improve type 2 diabetes management: baseline findings and six-month outcomes. BMC Public Health 2012;12(1):1-11.

46. Tropea P, Schlieter H, Sterpi I, Judica E, Gand K, Caprino M, et al. Rehabilitation, the great absentee of virtual coaching in medical care: scoping review. J Med Internet Res 2019;21(10):e12805.

47. Milne-Ives M, de Cock C, Lim E, Shehadeh MH, de Pennington N, Mole G, et al. The Effectiveness of Artificial Intelligence Conversational Agents in Health Care: Systematic Review. J Med Internet Res 2020;22(10):e20346.

48. Gong E, Baptista S, Russell A, Scuffham P, Riddell M, Speight J, et al. My Diabetes Coach, a mobile application-based interactive conversational agent to support type 2 diabetes self-management: A randomized effectiveness-implementation trial. Forthcoming 2021.

49. Kramer LL, Ter Stal S, Mulder BC, de Vet E, van Velsen L. Developing Embodied Conversational Agents for Coaching People in a Healthy Lifestyle: Scoping Review. J Med Internet Res 2020;22(2):e14058.

50. Baptista S, Wadley G, Bird D, Harris M, Riddell M, Russell A, et al. Acceptability of an embodied conversational agent for type 2 diabetes self-management education and support via a smartphone app: a mixed-methods study. Diabetes Technol Ther 2020;22:A221-2.

51. Gao L, Maddison R, Rawstorn J, Ball K, Oldenburg B, Chow C, et al. Protocol: Economic evaluation protocol for a multicentre randomised controlled trial to compare Smartphone Cardiac Rehabilitation, Assisted self-Management (SCRAM) versus usual care cardiac rehabilitation among people with coronary heart disease. BMJ Open
2020;10(8).

52. The Heart Foundation. Australian heart disease statistics [Internet]. The Heart Foundation. 2020 [cited 2020 Apr 22]. Available from: https://www. heartfoundation.org.au/about-us/what-we-do/ heart-disease-in-australia/australian-heart-disease-statistics

53. Böhmer M, Hecht B, Schöning J, Krüger A, Bauer G. Falling asleep with angry birds, facebook and kindle: a large scale study on mobile application usage. In: Proceedings of the 13th Conference on Human-Computer Interaction with Mobile Devices and Services, Mobile HCI; 2011. p. 47-56.

54. Meadows R, Hine C, Suddaby E. Conversational agents and the making of mental health recovery. Digit Health 2020;6:2055207620966170.

55. Sebastian J, Richards D. Changing stigmatizing attitudes to mental health via education and contact with embodied conversational agents. Comput Hum Behav 2017;73:479-88.

56. Open source conversational AI [Internet]. Rasa. 2020 [cited 2021 Feb 2]. Available from: https:// rasa.com/

57. Gong E, Baptista S, Russell A, Scuffham P, Riddell M, Speight J, et al. My Diabetes Coach, a Mobile App-Based Interactive Conversational Agent to Support Type 2 Diabetes Self-Management: Randomized Effectiveness-Implementation Trial. J Med Internet Res 2020 Nov 5;22(11):e20322.

58. Mental Health Chatbot [Internet]. Woebot Health. [cited 2021 Feb 2]. Available from: https://woebothealth.com/

59. Liao K, Liu Q, Wei Z, Peng B, Chen Q, Sun W, et al. Task-oriented Dialogue System for Automatic Disease Diagnosis via Hierarchical Reinforcement Learning. ArXiv Prepr ArXiv200414254. 2020.

Correspondence to:

Tilman Dingler

Tel.: + 61413873517

E-mail: tilman.dingler@unimelb.edu.au 